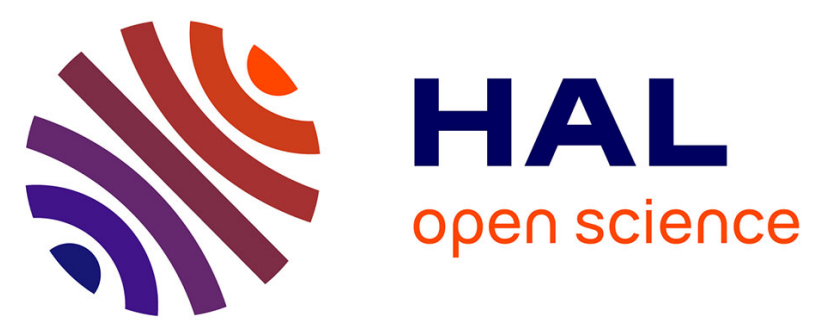

\title{
Further to the Left: Stress-Induced Increase of Spatial Pseudoneglect During the COVID-19 Lockdown
}

Federica Somma, Paolo Bartolomeo, Federica Vallone, Antonietta Argiuolo, Antonio Cerrato, Orazio Miglino, Laura Mandolesi, Maria Clelia Zurlo, Onofrio Gigliotta

\section{To cite this version:}

Federica Somma, Paolo Bartolomeo, Federica Vallone, Antonietta Argiuolo, Antonio Cerrato, et al.. Further to the Left: Stress-Induced Increase of Spatial Pseudoneglect During the COVID-19 Lockdown. Frontiers in Psychology, 2021, 12, pp.573846. 10.3389/fpsyg.2021.573846 . hal-03179135

\section{HAL Id: hal-03179135 \\ https://hal.sorbonne-universite.fr/hal-03179135}

Submitted on 24 Mar 2021

HAL is a multi-disciplinary open access archive for the deposit and dissemination of scientific research documents, whether they are published or not. The documents may come from teaching and research institutions in France or abroad, or from public or private research centers.
L'archive ouverte pluridisciplinaire HAL, est destinée au dépôt et à la diffusion de documents scientifiques de niveau recherche, publiés ou non, émanant des établissements d'enseignement et de recherche français ou étrangers, des laboratoires publics ou privés. 
OPEN ACCESS

Edited by:

Gianluca Castelnuovo, Catholic University of the Sacred Heart, Italy

Reviewed by:

Christopher S. Y. Benwell, University of Dundee, United Kingdom Anita D'Anselmo,

University of Bologna, Italy Sebastian Ocklenburg, Ruhr University Bochum, Germany

*Correspondence: Onofrio Gigliotta onofrio.gigliotta@unina.it

${ }^{\dagger}$ Deceased

Specialty section: This article was submitted to Psychopathology,

a section of the journal

Frontiers in Psychology

Received: 19 June 2020 Accepted: 18 January 2021 Published: 24 February 2021

Citation:

Somma F, Bartolomeo $P$ Vallone $F$, Argiuolo A, Cerrato A, Miglino O, Mandolesi L, Zurlo MC and Gigliotta O (2021) Further to the Left:

Stress-Induced Increase of Spatial Pseudoneglect During the COVID-19

Lockdown.

Front. Psychol. 12:573846. doi: 10.3389/fpsyg.2021.573846

\section{Further to the Left: Stress-Induced Increase of Spatial Pseudoneglect During the COVID-19 Lockdown}

\author{
Federica Somma1, Paolo Bartolomeo², Federica Vallone ${ }^{3,4}$, Antonietta Argiuolo', \\ Antonio Cerrato ${ }^{1+}$, Orazio Miglino ${ }^{1,5}$, Laura Mandolesi ${ }^{4}$, Maria Clelia Zurlo ${ }^{3}$ and \\ Onofrio Gigliotta ${ }^{1 *}$
}

' Natural and Artificial Cognition Laboratory, Department of Humanities, University of Naples Federico II, Naples, Italy, 2 Sorbonne Université, Inserm U 1127, CNRS UMR 7225, Paris Brain Institute, ICM, Hôpital de la Pitié-Salpêtrière, Paris, France, ${ }^{3}$ Dynamic Psychology Laboratory, Department of Political Sciences, University of Naples Federico II, Naples, Italy, ${ }^{4}$ Department of Humanities, University of Naples Federico II, Naples, Italy, ${ }^{5}$ Institute of Cognitive Sciences and Technologies, National Research Council, Rome, Italy

Background: The measures taken to contain the coronavirus disease 2019 (COVID19) pandemic, such as the lockdown in Italy, do impact psychological health; yet, less is known about their effect on cognitive functioning. The transactional theory of stress predicts reciprocal influences between perceived stress and cognitive performance. However, the effects of a period of stress due to social isolation on spatial cognition and exploration have been little examined. The aim of the present study was to investigate the possible effects and impact of the COVID-19 pandemic on spatial cognition tasks, particularly those concerning spatial exploration, and the physiological leftward bias known as pseudoneglect. A right-hemisphere asymmetry for spatial attention processes crucially contributes to pseudoneglect. Other evidence indicates a predominantly righthemisphere activity in stressful situations. We also analyzed the effects of lockdown on coping strategies, which typically show an opposite pattern of hemispheric asymmetry, favoring the left hemisphere. If so, then pseudoneglect should increase during the lockdown and be negatively correlated with the efficacy of coping strategies.

Methods: One week before the start of the lockdown due to COVID-19 in Italy (T1), we had collected data from a battery of behavioral tests including tasks of peri-personal spatial cognition. During the quarantine period, from late April to early May 2020 (T2), we repeated the testing sessions with a subgroup of the same participants (47 righthanded students, mean age $=20, S D=1.33$ ). At both testing sessions, participants performed digitized neuropsychological tests, including a Cancellation task, Radial Arm Maze task, and Raven's Advanced Progressive Matrices. Participants also completed a newly developed COVID-19 Student Stress Scale, based on transactional models of stress, and the Coping Orientation to Problems Experienced-New Italian Version (COPE-NIV) to assess coping orientation.

Results: The tendency to start cancelation from a left-sided item, to explore first a leftsided arm of the maze, and to choose erroneous response items on the left side of the page on Raven's matrices increased from T1 to T2. The degree of pseudoneglect 
increment positively correlated with perceived stress and negatively correlated with Positive Attitude and Problem-Solving COPE-NIV subscales.

Conclusion: Lockdown-related stress may have contributed to increase leftward bias during quarantine through a greater activation of the right hemisphere. On the other hand, pseudoneglect was decreased for better coping participants, perhaps as a consequence of a more balanced hemispheric activity in these individuals.

Keywords: cognition, psychology, stress, pseudoneglect, coronavirus, quarantine, pandemic

\section{INTRODUCTION}

Coronavirus disease 2019 (COVID-19) broke into a worldwide pandemic (World Health Organization, 2020) at the beginning of 2020. At the time of writing, there are more than 7.5 million confirmed cases throughout 215 countries, with more than 400,000 deaths. Italy was the first European Union (EU) country to be hit by a dramatic COVID-19 outbreak, with a quick and heavy impact on public physical and psychological health. Millions of people have experienced an abrupt change in their lives, due not only to the spreading of the illness but also to the measures put in place to prevent the contagion and limit the outbreak. On March 9, 2020, the Italian government imposed a national quarantine and several consequent lockdown restrictions, which ended on May 4, 2020, for some activities and on May 18, 2020, for others. Thus, the Italian quarantine lasted 70 days.

Brooks et al. (2020) examined previous research on the psychological impact of quarantine and reported a high prevalence of symptoms of psychological distress and disorder: especially low mood and irritability, but also emotional disturbance, depression, stress, insomnia, post-traumatic stress symptoms, etc. Moreover, they found that the effects of security measures due to a pandemic can affect not only short-term but also long-term psychological state (Brooks et al., 2020). Poor sleep quality, for example, can affect mental health (Gehrman et al., 2013; Franceschini et al., 2020) and alter emotional and cognitive functioning (Altena et al., 2020). As emphasized by the American Psychological Association (Novotney, 2020), social isolation can trigger several health risks. Feeling isolated can lead to poor sleep, poor cardiovascular health, depressive symptoms, and impaired executive function. These effects tend to impair the ability to stay focused, emotional control, retrieval of information, and the capacity to follow directions. Even brief periods of loneliness and isolation can have negative consequences on both physical and mental well-being (Cacioppo and Patrick, 2008).

Restrictions could affect different life domains among students, inducing specific perceived stressors related to academic studying, relationships with university colleagues, relationships with professors, social isolation, risk of contagion, relationships with relatives, and sexual life (Zurlo et al., 2020). Moreover, restrictions could also affect physical activity, socializing (except virtual social media), proper nutrition, and good quality of sleep. These restrictions were likely to result in increased stress and psychological disease (Mandolesi et al., 2018; Brooks et al., 2020; Zurlo et al., 2020) as well as in recourse to coping strategies to deal with it. However, less is known about the potential impact of quarantine on spatial cognition, a heterogenous set of processes incorporating spatial memory (Mandolesi et al., 2009b; Sorrentino et al., 2019), egocentric and allocentric representation and mapping abilities (Klatzky, 1998; Foti et al., 2020), and visuoperceptive abilities, including spatial attention (Newcombe and Huttenlocher, 2007; Bartolomeo et al., 2012; Newcombe, 2018; Bartolomeo and Malkinson, 2019; Bartolomeo, 2020).

The transactional theory of stress predicts a reciprocal influence between perceived stress and cognitive performance and underlines the key role played by individual differences, such as coping strategies, in influencing this relationship (Lazarus and Folkman, 1984; Matthews et al., 2000). The individual adaptation process to a significant source of stress (such as the current COVID-19 pandemic lockdown) consists of appraisals of primary control (i.e., perceived possibilities to modify the situation to reduce its negative impact) and secondary control (i.e., perceived possibilities to modify the appraisal of circumstances to achieve a positive adjustment).

Thus, similar sources of stress may have a different subjective impact. Stress is a dynamic concept, depending on the constant interplay between individual and situational factors that reciprocally influence each other, and the potential efficacy of the different coping strategies adopted to deal with perceived stress and to enhance adjustment is strongly situation-specific and related to the interaction between the individuals and the situations (Zurlo et al., 2013, 2019). From this perspective, in particular, a perceived lack of controllability can lead to lower levels of performance (Matthews and Campbell, 2009).

Little is known about the effects of a period of stress (such as quarantine and social isolation) on spatial cognition. Animals being exposed to chronic stress show impaired exploratory behavior (Brydges et al., 2012; ter Horst et al., 2012; van der Kooij et al., 2018). Rats exposed to chronic stress in their early life show atypical leftward asymmetry in turning behavior (Mundorf et al., 2020). In particular, stress might play different roles at different stages of development: early exposition might lead to structural brain changes, whereas later exposition might modulate functional aspects (Berretz et al., 2020).

Gruzelier and Phelan (1991) found that stress was able to shift the hemispheric balance in a divided visual field lexical task toward the left visual field in a sample of medical students. Richardson and VanderKaay Tomasulo (2011) induced stress in human participants by using the frustrating Star Mirror Tracing Task and found slower spatial responses in a navigation task and a perspective taking task, as compared with non-stressed control 
participants. However, Schwabe et al. (2007) found no evidence of an effect of stress on spatial learning, and Duncko et al. (2007) found improved performance on a virtual navigation task after hand immersion in ice water (cold pressor stress).

Directional spatial effects offer a possibility to quantify the effects of stress on spatial cognition. A basic, evolutionarily conserved pattern of asymmetry sees the right hemisphere taking control of responses to novel, unpredicted and potentially dangerous changes in the environment (Compton et al., 2000; Vallortigara and Versace, 2017; Bartolomeo and Malkinson, 2019). Another, well-known pattern of asymmetry favoring the human right hemisphere concerns the fronto-parietal brain networks important for orienting and control of spatial attention (Corbetta et al., 2008; Bartolomeo and Malkinson, 2019). A relative hyperactivity of right-hemisphere attention networks might push spatial attention leftward. This directional attention bias contributes to a small, physiological leftward bias in spatial processing (Toba et al., 2011), labeled pseudoneglect (Bowers and Heilman, 1980). Pseudoneglect can manifest itself during the bisection of horizontal lines, as a small leftward deviation of the subjective midpoint (Jewell and McCourt, 2000) or as a bias to start visual search from a left-sided item (Gigliotta et al., 2017).

Evidence on structural and functional brain asymmetries regarding attention networks and stress response (both involving the right hemisphere, see Ocklenburg et al., 2016; Zach et al., 2016; Gigliotta et al., 2017) led us to hypothesize a relation between stress and pseudoneglect. Specifically, two predictions were made: (1) higher level of stress should increase the magnitude of pseudoneglect and (2) effective coping strategies that may preferentially reduce right-hemisphere activation (Lindauer et al., 2008) should reflect in a lower magnitude of pseudoneglect. Just before the beginning of the COVID19 lockdown, we had assessed visuospatial performances in a group of Italian university students. Thus, we had the unique opportunity to test our predictions by comparing students' performances before and during the lockdown.

\section{MATERIALS AND METHODS}

\section{Participants}

Throughout the month of February 2020, before the Italian lockdown, we conducted data collection sessions on peripersonal spatial cognition tasks. The data collection ended on March 2, exactly 1 week before the start of the lockdown due to COVID-19 in Italy. We therefore decided to perform a second data collection with the same participants during the quarantine period. Specifically, the session lasted 2 weeks from late April to early May.

Before the beginning of the lockdown, 102 Psychology and Philosophy students (81 females) of the University of Naples Federico II aged between 18 and 26 years $($ mean $=19.5$, $\mathrm{SD}=1.5$ ) voluntarily enrolled in the first experimental session. Selection criteria for participants' recruitment included normal or corrected-to-normal vision. Students were contacted later on during quarantine, and 55 out of 102 students agreed to participate in the second session. Seven out of 55 participants were excluded because they reported left hand preference. Left-handers were excluded because of evidence of higher performance variability on visuospatial tasks (see, for example, Sampaio and Chokron, 1992) and of decreased pseudoneglect effects (Jewell and McCourt, 2000). One additional participant did not conclude the session because of technical problems. The final sample consisted of 47 right-handed students, aged between 18 and 24 years $($ mean $=20, S D=1.33), 41$ females and 6 males.

Written informed consent was obtained from all participants. The study was approved by the Local Ethics Committee of the University of Naples Federico II (protocol number: 12/2020) and was carried out in accordance with the Declaration of Helsinki.

\section{Measures \\ Neuropsychological Tests \\ Cancellation Task}

In the present study, we administered a digitized Cancellation task developed by Gigliotta et al. (2017). Each trial starts with participants touching (or clicking on) a green button located at the center of the screen. Participants are then presented with five round red stimuli randomly arranged on an electronic screen. They have to cancel all the stimuli as fast as possible with a stylus pen touch or a mouse click (depending on the user interface). The canceled item changes in color to a brighter nuance of red. Thirty trials were administered, with randomly different spatial disposition of targets.

\section{Radial Arm Maze Task}

The Radial Arm Maze (RAM; Olton and Samuelson, 1976) consists of a central area with identical radiating arms. It is extensively used to assess the spatial abilities of laboratory rodents and human participants (Overman et al., 1996; Mandolesi et al., 2009a,b; Foti et al., 2011, 2020). The aim is to recover rewards hidden at the end of each arm. Different strategies can be implemented (for example, visit a specific sequence of arms, adjacent, opposite, or alternating, etc.). We used a digitized version (Mandolesi and Gigliotta, submitted) whereby participants control a ladybug, positioned in the center of the labyrinth, along the arms to retrieve hidden ladybugs placed at the end of each arm. There were six trials, with a time limit of $60 \mathrm{~s}$ per trial. The number of arms gradually increased over trials from 3 to 8 arms. In the present work, we analyzed results from the 8 -arm maze, the condition with the highest spatial resolution.

\section{Raven's Advanced Progressive Matrices}

The Raven's Advanced Progressive Matrices (APM; Raven et al., 1962) are used to assess non-verbal and "fluid" intelligence and require the direct analysis, construction, and integration of a series of visual items. Raven's matrices questions consist of visual geometric designs with a missing piece. Participants are asked to choose the missing piece between eight alternatives, arranged along four vertical columns disposed from the left to the right of the page below the test image. A digital version of the Raven's APM was administered in the present study, for which the matrices were transposed on Google Modules. Only set I (12 items with 8 possible responses) was administered. 
Costa et al. (1969) administered the Raven's APM to patients suffering from left neglect after right-hemisphere damage and assessed the spatial side (left or right) of the error responses. The results showed that patients tended to erroneously choose right-sided items. Colombo et al. (1976) administered Raven's APM to patients with left and right brain injuries and found that patients tended to prefer ipsilesional candidate items. This position preference was especially evident in patients with righthemisphere damage. This evidence, suggesting that spatial biases can influence performance on the Raven's APM, incited us to employ such a space-based assessment in the present setting.

\section{Questionnaires}

\section{The COVID-19 Student Stress Questionnaire}

The COVID-19 Student Stress Questionnaire (CSSQ; Zurlo et al., 2020) was specifically developed to assess university students' perceived stress during the COVID-19 pandemic lockdown. It consists of 7 items on a 5-point Likert scale ranging from 0 ("not at all stressful") to 4 ("extremely stressful"). For the purpose of instrument design, perceived stress was operationalized based on transactional models of stress (Lazarus and Folkman, 1984). Each item was developed to cover different domains that could have been subject to variations due to the COVID-19 pandemic lockdown and, therefore, that may be potentially perceived as sources of stress (i.e., risk of contagion; social isolation; relationship with relatives; relationship with colleagues; relationship with professors; academic studying; couple's relationship, intimacy, and sexual life). The scale provides a Global Stress score ranging from 0 to 28 . The CSSQ was developed and tested in a sample of 514 Italian university students, and it was confirmed to be a valid and reliable measure. The Global Stress score revealed significant correlations, in the expected directions, with measures of Anxiety $(r=0.55, p<0.01)$, Depression $(r=0.56, p<0.01)$, and Somatization $(r=0.39, p<0.01)$, as assessed by means of the Symptom Checklist-90-Revised (SCL-90-R; Prunas et al., 2010). The questionnaire revealed a satisfactory internal consistency $($ Cronbach's alpha $=0.71)$.

The results of the CSSQ scale validation study highlighted the presence of three significant factors, which the authors labeled as: 1) "Relationships and Academic Life," which comprised the four items covering perceived stress related to relationships with relatives, relationships with colleagues, relationships with professors, and academic studying; 2) "Isolation," which comprised the two items exploring perceived stress related to social isolation and changes in sexual life due to the containment measures; and 3) "Fear of Contagion," which comprised the item assessing perceived stress related to the risk of infection. Therefore, we decided to analyze any relationships between the increase in left bias and the stress measured through the CSSQ scale.

\section{Coping Orientation to Problems Experienced-New Italian Version (COPE-NIV; Sica et al., 2008)}

The questionnaire consists of 60 items on a 5-point Likert scale ranging from 1 ("I usually don't do this at all") to 4 ("I usually do this a lot") divided into five subscales: Seeking
Social Support (12 items covering strategies centered on seeking support for instrumental or emotional reasons and focusing on and venting of emotions; Cronbach's $\alpha=0.88$ ), Avoiding (16 items covering strategies centered on detaching, denial, humor, alcohol and drug disengagement, behavioral disengagement, and mental disengagement; Cronbach's $\alpha=0.70$ ), Positive Attitude (12 items covering strategies centered on positive reinterpretation and restraint coping; Cronbach's $\alpha=0.76$ ), Problem Solving (12 items covering strategies centered on suppression of competing activities, planning, and active coping; Cronbach's $\alpha=0.83$ ), and Turning to Religion (8 items covering strategies centered on seeking comfort in religious and spiritual practices; Cronbach's $\alpha=0.85$ ).

\section{Procedure}

\section{Pre-Lockdown Session (T1)}

The first experimental session took place in a quiet room of the University of Naples Federico II. In the room, there was a large table with chairs around it; on the table, there were an 8-inch tablet to be used by participants and a computer in front of the experimenter. Participants sat in front of the experimenter. The total time to complete all tests was around $20 \mathrm{~min}$.

The first session test battery we administered included, among other tests, the Cancellation task, the RAM task, and the Raven's APM task. The Cancellation task and the RAM task were administered by means of specific software running on an 8inch tablet and performed using a stylus pen to interact with the screen. Participants were comfortably seated with a viewing distance of $\sim 40 \mathrm{~cm}$, and the tablet was placed on the table in front of them in a vertical position.

A digital version of the Raven's APM was first administered, for which the matrices were transposed on Google Modules. Participants used a 14-inch PC and a mouse to perform the APM. Then, the Cancellation task was administered on the tablet. The instructions were as follows: "As soon as you select the green button with the pen you will see little circles, which you will have to select all in the shortest possible time. If you happen to touch the white screen it will turn black for a moment, then you go on." Finally, the digitized RAM task was performed by participants. The instructions were as follows: "The objective of this task is to explore all the arms of the mazes, dragging the ladybug there, and find the ladybugs hidden under the jars within $60 \mathrm{~s}$. Remember that you must always go to the center before moving from one arm to another. The first item is for practice."

\section{Lockdown Session (T2)}

After about 2 months from the start of the lockdown, we contacted the participants of the first experimental session. A subgroup of 47 students agreed to participate in the second experimental session. The procedure consisted in the administration of the questionnaires, then in the online administration of the Cancellation task, and the digitized RAM task to the participants, through Microsoft ${ }^{\circledR}$ Teams, a unified communication and collaboration platform that combines chat, teleconferencing, content sharing, and application integration.

Participants were asked to fill out the questionnaires on Microsoft ${ }^{\circledR}$ Forms, an online survey maker software. A few 
days after completing the questionnaires, one experimenter started the cognitive test sessions and carried out the online meetings on Microsoft ${ }^{\circledR}$ Teams platform, with each student separately. The experimenter first explained the test procedure methods, ensuring that both network connections were working properly. The software implementing the spatial tasks ran on the experimenter's computer. After giving the same task instructions as in the pre-lockdown session, the experimenter activated the Microsoft ${ }^{\circledR}$ Teams platform's screen sharing mode, so that the participants had the control of the experimenter's screen and were able to carry out the tasks. Thus, there were minor differences in user interface between T1 (touch stylus used for the Cancellation task and the RAM task) and T2, when the mouse was instead used for all tests.

\section{Parameters}

In both the experimental sessions, the following parameters were analyzed.

For the Cancellation task, we first defined the center of the display as 0 , so that the values of the $\mathrm{X}$ pixel coordinates assumed a negative sign for the left side of the screen and a positive sign for the right side. Then, we calculated the average position on the $\mathrm{x}$-axis of the first canceled stimulus for each participant (see Gigliotta et al., 2017, for a detailed description of the procedure). In order to assess potential differences in spatial bias before (T1) and during (T2) the lockdown, we calculated the increment of leftward preference, in canceling the first stimuli, from T1 to T2.

For the RAM task, we defined as 0 the center of the display. The values of the $\mathrm{X}$ pixel coordinates were negative for the left side of the screen and positive for the right side. We focused on the performance on the 8-arm maze, which offered participants the largest number of potential exploration strategies. We assessed the coordinates of the first explored arm for the 8-arm maze, as well as the spatial sequence of the visited arms.

For Raven's APM, in addition to the test scores, we obtained a measure of position preference (see Costa et al., 1969), by assessing the location in space (left or right) of the error responses chosen below the target figure of each matrix. Therefore, we calculated the average number of items erroneously chosen for each side of space, among the four left-sided and the four rightsided alternatives.

\section{RESULTS}

Data analysis was run on JASP (https://jaspstats.org/), version 0.12.2.

\section{Cancellation Task}

First, we investigated the presence of a lateralization of the first canceled stimulus in the Cancellation task: results showed a leftbiased distribution of the first canceled stimulus both for T1 (Wilcoxon-Mann-Whitney two-tailed test, $Z=-103, p<0.001$; mean $=-60.1$, SD $=62.33$ ) and for T2 (Wilcoxon-MannWhitney two-tailed test, $Z=-18, p<0.001$; mean $=-83.46$, $\mathrm{SD}=49.97$ ), thus confirming the previously reported tendency to start the visual search from a left-sided target (pseudoneglect) on this task (Gigliotta et al., 2017).

Then, a repeated measure ANOVA was conducted on the spatial $\mathrm{X}$ coordinates of the first canceled stimulus for each trial in $\mathrm{T} 1$ and $\mathrm{T} 2$, to evaluate potential lockdown-induced changes in patterns of spatial exploration. The independent variable was the time of testing (T1, T2); the dependent variable was the coordinate (in pixels) of the first canceled stimulus. Figure 1 shows that, on average, the first canceled stimulus at T2 was 23 pixels further to the left (mean $=-83.46$ pixels, $\mathrm{SD}=49.97$ ) as compared with its position at $\mathrm{T} 1$ [mean $=-60.10$, $\mathrm{SD}=62.33, F(1,46)=6.10, p=0.017$, with a moderate sample size effect, $\left.\eta^{2}=0.117\right]$.

To further assess the potential relationship between stress and magnitude of pseudoneglect, we conducted a two-tailed Pearson's correlation analysis between the results of the CSSQ and the pseudoneglect increment at T2 from T1. The null hypothesis was that the two variables are not related in our sample; conversely, the alternative hypothesis was that the stress and the magnitude of pseudoneglect are related. The results showed a significant correlation between the CSSQ scale and the leftward biased exploration of the space $(r=0.407, p=0.004)$, so we could accept the alternative hypothesis and reject the null one: particularly, as stress levels increase, the exploration bias to the left seems to be also accentuated (Figure 2).

A second two-tailed Pearson's correlation analysis investigated the potential relationship between coping (assessed through the five subscales of the COPE-NIV) and pseudoneglect increment at T2. The results showed a significant correlation between 2 out of 5 COPE-NIV subscales (Positive Attitude and Problem Solving) and lower leftward biased exploration of the space

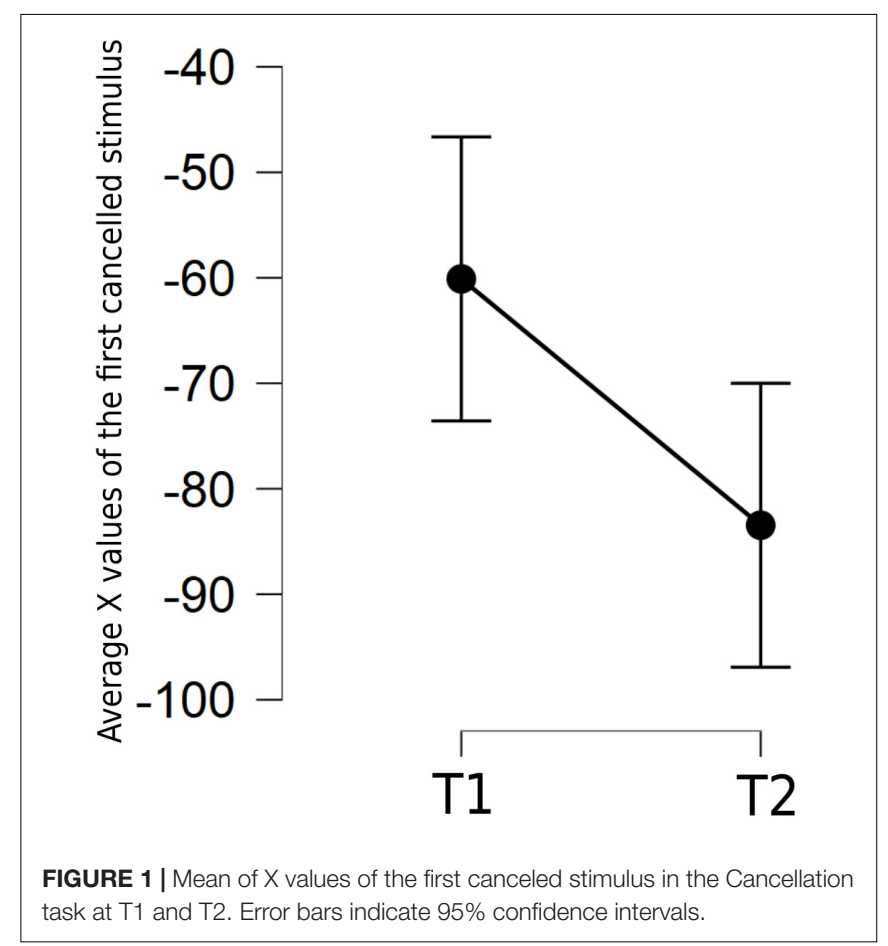




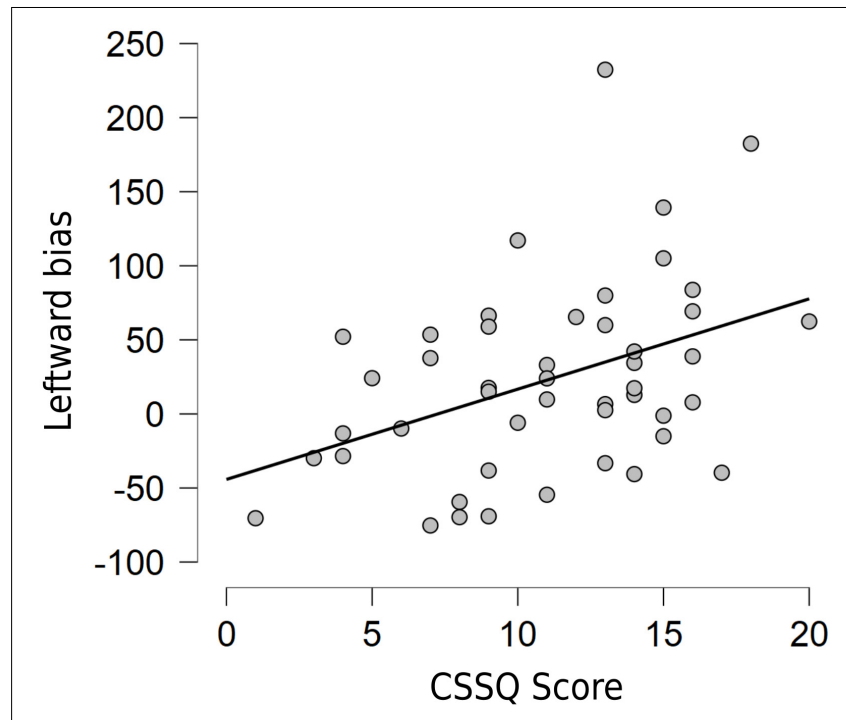

FIGURE 2 | Relationship between the CSSQ scale global score and leftward bias variation in the Cancellation task from $\mathrm{T} 1$ to $\mathrm{T} 2$.

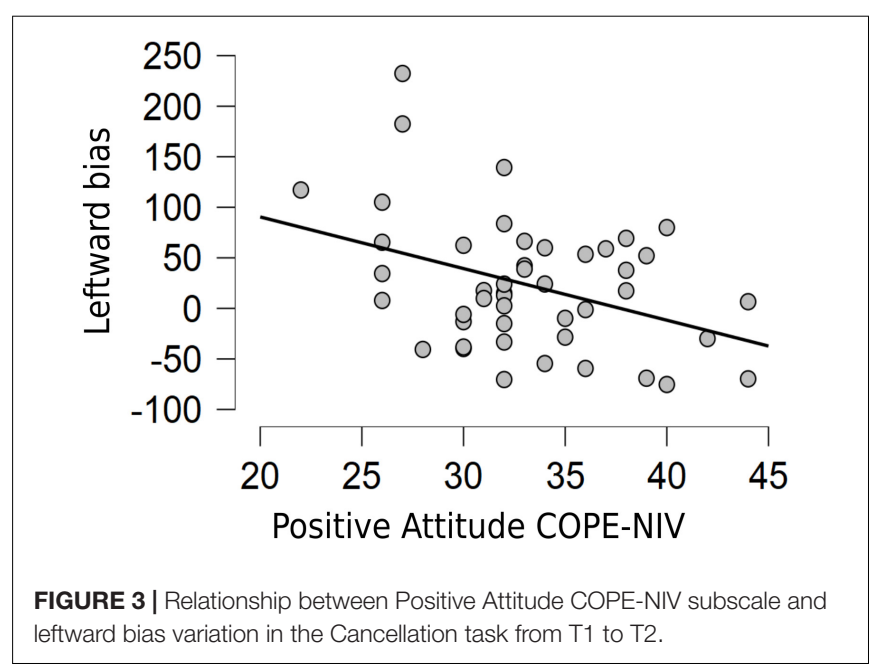

( $r=-0.385, p=0.008$ and $r=-0.308, p=0.037$, respectively; see Figures 3, 4). This correlation indicates that pseudoneglect decreases with increasing active coping strategies. Instead, no significant correlation resulted between the other COPE-NIV subscales, Seeking Social Support $(r=0.100, p=0.507)$, Avoiding ( $r=-0.072, p=0.633)$, and Turning to Religion $(r=-0.116$, $p=0.445)$.

\section{Digitized RAM Task}

We investigated the lateralization of the first explored arm of the RAM and verified if there was a significant variability of the lateralization between $\mathrm{T} 1$ and $\mathrm{T} 2$. A repeated measures ANOVA on the $\mathrm{x}$ coordinates of the first arm (of the 8-arm maze) chosen indicated that participants tended to start exploration from a leftsided arm at T2 (mean $=-23.04, \mathrm{SD}=112.74$ ), whereas they preferred to start from a right-sided arm at $\mathrm{T} 1$ [mean $=21.62$, $\left.\mathrm{SD}=110.47, \mathrm{~F}(1,46)=5.31, p=0.03, \eta^{2}=0.103\right]$ (Figure 5).
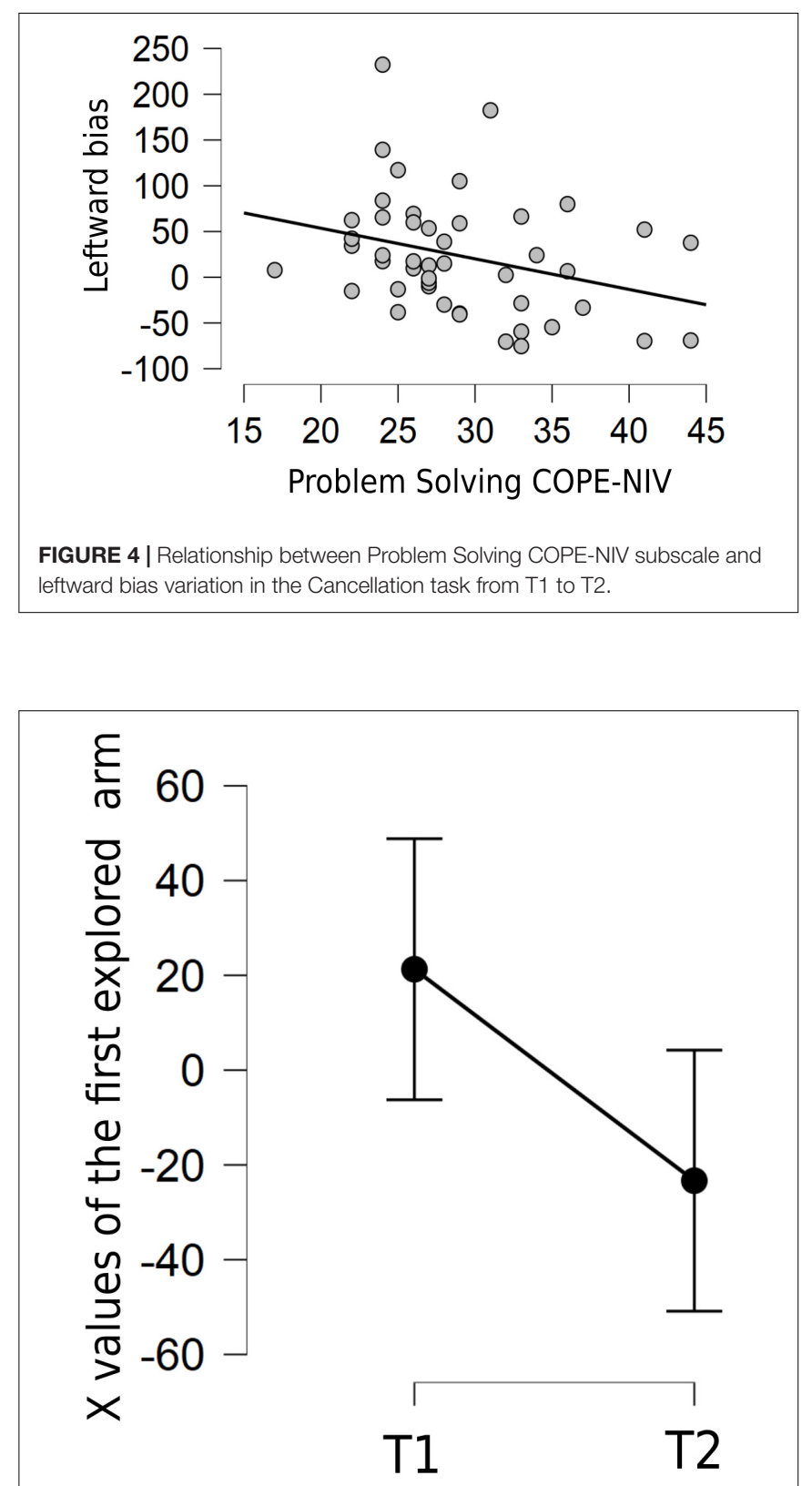

FIGURE 5 | Mean of $X$ values of the first explored arm on the digitized RAM task at T1 and T2. Error bars indicate 95\% confidence intervals.

Then, to assess a potential relationship between stress/coping and the deviation of the maze exploration to the left side at T2, a two-tailed Pearson's correlation analysis between the results of the CSSQ/COPE-NIV and the laterality variation at T2 from T1 was conducted. The results do not show any significant correlation of the variation in lateralization, neither with the global score of the CSSQ scale $(r=-0.173, p=0.246)$ nor with the CSSQ subscale scores, nor with the 5 COPE-NIV subscales, such as Positive Attitude and Problem Solving coping strategies ( $r=-0.092, p=0.545$ and $r=-0.019, p=0.899$, respectively). 


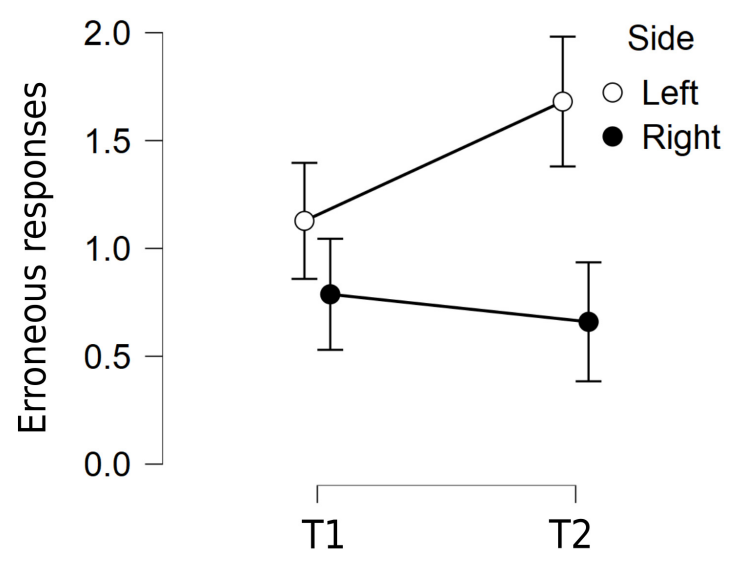

FIGURE 6 | Erroneous responses to the Raven's Advanced Progressive Matrices at T1 and T2 for the right and left sides. Error bars indicate 95\% confidence intervals.

\section{Raven's APM}

Participants obtained scores in the normal range both at $\mathrm{T} 1$ $($ mean $=10.085, \mathrm{SD}=1.851)$ and at $\mathrm{T} 2($ mean $=9.745$, $\mathrm{SD}=1.750$ ). There was no significant effect of time of testing on accuracy [repeated measures ANOVA on the correct answers, $F(1,46)=2.012, p=0.163]$.

To assess changes in position preference for erroneous responses (Costa et al., 1969), we conducted a 2 (period of testing: T1, T2) $\times 2$ (error side: right, left) repeated measures ANOVA. There was a main effect of error side: $F(1,46)=22.41$, $p<0.001, \eta^{2}=0.14$, because participants showed a bias to choose a left-sided item (mean $=1.40, \mathrm{SD}=1.30)$ over a right-sided one (mean $=0.72, \mathrm{SD}=1.01$ ). Time of testing approached significance $(p=0.06)$, because participants tended to make more errors during quarantine (mean $=1.17$ ) than before it (mean $=0.96$ ). Importantly, the two factors interacted $F(1,46)=4.91, p=0.032, \eta^{2}=0.036$, because the leftward bias increased during quarantine (Figure 6).

Finally, to assess a potential relationship between stress/coping and the deviation of Raven's errors to the left side at T2, a twotailed Pearson's correlation analysis between the results of the CSSQ/COPE-NIV and the laterality variation at T2 from T1 was conducted. The results do not show any significant correlation of the variation in lateralization, neither with the global score of the CSSQ scale $(r=-0.017, p=0.910)$ nor with the CSSQ subscale scores, nor with the 5 COPE-NIV subscales, such as Positive Attitude and Problem Solving coping strategies $(r=-0.148$, $p=0.327$ and $r=-0.079, p=0.604$, respectively).

\section{DISCUSSION AND CONCLUSION}

The aim of this research was to assess whether the stressful conditions experienced by students during the harsh quarantine measures taken in Italy (a country strongly hit by COVID-19) had any influence on their spatial cognition abilities. The results indicated several indices of such an influence.
Specifically, we found a significant leftward shift in three tasks tapping on spatial abilities from T1 (pre-lockdown) to T2 (circa after 2 months of harsh quarantine). The tasks were (a) the Cancellation task, (b) the digitized RAM task, and (c) the Raven's APM task. Importantly, there were no changes in the general accuracy on the Raven's APM task, a test of general nonverbal intelligence; the only time-related change was an increase of leftward spatial bias in the choice of an (erroneous) response item. Thus, the time-related changes we observed seem to be relatively specific to the spatial domain. A potential confound could be the mode of response used for the Cancellation task. Participants used a touch pen at T1 and a computer mouse at T2. However, a similar increase in leftward bias with time also occurred on the Raven's matrices, where participants always responded by using the mouse.

Were these changes in spatial bias really related to lockdowninduced stress? Evidence supporting this possibility comes from (1) the positive correlation between stress measured through the CSSQ scale and the increase of pseudoneglect during the lockdown and (2) the negative correlation that we observed between the increment of pseudoneglect and specific active coping strategies, which mirrored the positive correlations between pseudoneglect and time of testing on the Cancellation task (for the other tasks, we should take into account differences on the cognitive functions they rely on and different measures of stress). Individuals who were able to resort to positive attitude and problem-solving coping strategies displayed lesser leftward bias than those who obtained higher scores in perceived stress.

Our findings seem, therefore, in line with research underlining the impact of perceived stress on individuals' performance (Matthews et al., 2000; Matthews and Campbell, 2009). They also provide new evidence supporting the efficacy of the adoption of strategies centered on activity and positive reappraisal (Santarnecchi et al., 2018; Zurlo et al., 2019).

The neurobiological underpinnings of physiological leftward bias (pseudoneglect) are likely to rely on hemispheric asymmetries of attention networks (Corbetta et al., 2008; Bartolomeo and Malkinson, 2019). Shifts in line bisection strictly depend on activity in these fronto-parietal networks (Thiebaut de Schotten et al., 2005). For example, activity in the right ventral attention network seems to correlate with the effect of line length in pseudoneglect (Benwell et al., 2014). In a simulation study, Gigliotta et al. (2017) demonstrated that different patterns of asymmetries in artificial attention networks can lead to different levels of pseudoneglect in neuroagents (robots provided with a simulated brain) performing a Cancellation task similar to the one used here.

On the other hand, abundant evidence suggests a relation of stress-related mechanisms with the right hemisphere (Compton et al., 2000; Ocklenburg et al., 2016; Bartolomeo and Malkinson, 2019). Moreover, early life exposure to stress has been proposed as a determinant of psychiatric and neurodevelopmental diseases characterized by atypical brain asymmetries (Berretz et al., 2020). Finally, a recent study on turning behavior in rats highlighted a leftward shift in turning preferences in a group of animals exposed to stressful conditions during the early stage of their lives compared with a control group (Mundorf et al., 2020). 
Acute and chronic stress can thus affect lateralized behavior in humans and animals, as a result of higher right-hemisphere activation (Ocklenburg et al., 2016). In addition, stress in university students was found to increase connectivity in the attention networks, particularly in the right hemisphere (Soares et al., 2013). Over time, this functional modulation might translate into structural plastic changes. For example, Brem et al. (2020) performed white matter tractography on a group of volunteers after 520 days of confinement and found a general reduction in fractional anisotropy in the right temporo-parietal junction. Coping strategies, on the contrary, might be related to greater left hemisphere connectivity. Santarnecchi et al. (2018) found a positive correlation with the connectivity of the left angular gyrus of performance on the problem-solving subscale of the coping scale used in this study, the COPENVI.

The specific relation we found between stress and leftward bias is thus likely to depend at least in part on stress-induced increased activity of right-hemisphere attention networks. The right hemisphere may also facilitate stress hormone responses through the hypothalamic-pituitary-adrenal gland axis (Sullivan, 2004), whereas the left hemisphere structures, such as the medial prefrontal cortex, may increase resilience to stress and control its effects on social behavior (Lee et al., 2015).

The present study was conducted on a relatively limited participant sample $(N=47)$. As a consequence of the strict lockdown measures, the test conditions could not be fully controlled at T2. Despite these limitations, our results linking stress and leftward bias were consistent over several tests. More "ecological" tests of spatial cognition, closer to everyday life activities than the tests we employed here (Cerrato et al., 2019, 2020), may be useful to further assess these relationships. Studies in animals, as well as on simulated neurorobots (Broz et al., 2014; Gigliotta et al., 2015a,b, 2017; Pacella et al., 2017), might further

\section{REFERENCES}

Altena, E., Baglioni, C., Espie, C. A., Ellis, J., Gavriloff, D., Holzinger, B., et al. (2020). Dealing with sleep problems during home confinement due to the COVID-19 outbreak: practical recommendations from a task force of the European CBT-I Academy. J. Sleep Res. 29:e13052. doi: 10.1111/ jsr.13052

Bartolomeo, P. (2020). “Anatomy and disorders of the spatial attention system," in Encyclopedia of Behavioral Neuroscience: Second Edition, eds M. Thiebaut De Schotten and S. Della Sala (Amsterdam: Elsevier).

Bartolomeo, P., and Malkinson, S. T. (2019). Hemispheric lateralization of attention processes in the human brain. Curr. Opin. Psychol. 29, 90-96. doi: 10.1016/j.copsyc.2018.12.023

Bartolomeo, P., Thiebaut De Schotten, M., and Chica, A. B. (2012). Brain networks of visuospatial attention and their disruption in visual neglect. Front. Hum. Neurosci. 6:110. doi: 10.3389/fnhum.2012.00110

Benwell, C. S., Harvey, M., and Thut, G. (2014). On the neural origin of pseudoneglect: EEG-correlates of shifts in line bisection performance with manipulation of line length. Neuroimage 86, 370-380. doi: 10.1016/j. neuroimage.2013.10.014

Berretz, G., Wolf, O. T., Güntürkün, O., and Ocklenburg, S. (2020). Atypical lateralization in neurodevelopmental and psychiatric disorders: what is the role of stress? Cortex 125, 215-232. doi: 10.1016/j.cortex.2019. 12.019 illuminate the intimate mechanisms that link stress to spatial attention.

\section{DATA AVAILABILITY STATEMENT}

The raw data supporting the conclusions of this article will be made available by the authors, without undue reservation.

\section{ETHICS STATEMENT}

The studies involving human participants were reviewed and approved by the Local Ethics Committee of the University of Naples Federico II (protocol number: 12/2020). The patients/participants provided their written informed consent to participate in this study.

\section{AUTHOR CONTRIBUTIONS}

OG, LM, MZ, and PB contributed to the conception and design of the study. FS, FV, AA, and AC collected the data and wrote the first draft of the manuscript. OG performed the statistical analysis. All authors contributed to manuscript revision, and read and approved the submitted version.

\section{FUNDING}

This work has been supported by the University of Naples Federico II, the Institute for Cognitive Sciences and Technologies, Rome, the Program Investissements d'Avenir Grant ANR-10-IAIHU-06, and the Foundation Jérôme Lejeune (n. 1567, 2016B to LM).

Bowers, D., and Heilman, K. M. (1980). Pseudoneglect: effects of hemispace on a tactile line bisection task. Neuropsychologia 18, 491-498. doi: 10.1016/00283932(80)90151-7

Brem, C., Lutz, J., Vollmar, C., Feuerecker, M., Strewe, C., Nichiporuk, I., et al. (2020). Changes of brain DTI in healthy human subjects after 520 days isolation and confinement on a simulated mission to Mars. Life Sci. Space Res. 24, 83-90. doi: 10.1016/j.lssr.2019.09.004

Brooks, S. K., Webster, R. K., Smith, L. E., Woodland, L., Wessely, S., Greenberg, N., et al. (2020). The psychological impact of quarantine and how to reduce it: rapid review of the evidence. Lancet 395, 912-920. doi: 10.1016/S0140-6736(20) 30460-8

Broz, F., Nehaniv, C. L., Belpaeme, T., Bisio, A., Dautenhahn, K., Fadiga, L., et al. (2014). The ITALK project: a developmental robotics approach to the study of individual, social, and linguistic learning. Top. Cogn. Sci. 6, 534-544. doi: 10.1111/tops.12099

Brydges, N. M., Hall, L., Nicolson, R., Holmes, M. C., and Hall, J. (2012). The effects of juvenile stress on anxiety, cognitive bias and decision making in adulthood: a rat model. PLoS One 7:e0048143. doi: 10.1371/journal.pone.00 48143

Cacioppo, J. T., and Patrick, W. (2008). Loneliness: Human Nature and the Need for Social Connection. New York, NY: W.W. Norton \& Co.

Cerrato, A., Pacella, D., Palumbo, F., Beauvais, D., Ponticorvo, M., Miglino, M., et al. (2020). E-TAN, a technology-enhanced platform with tangible objects for the assessment of visual neglect: a multiple single-case study. 
Neuropsychol. Rehabil. [Epub ahead of print]. doi: 10.1080/09602011.2020.17 62671

Cerrato, A., Ponticorvo, M., Gigliotta, O., Bartolomeo, P., and Miglino, O. (2019). "The assessment of visuospatial abilities with tangible interfaces and machine learning," in Understanding the Brain Function and Emotions, eds J. Ferrández Vicente, J. Álvarez-Sánchez, F. de la Paz López, J. Toledo Moreo, and H. Adeli (Spain: IWINAC), 78-87.

Colombo, A., De Renzi, E., and Faglioni, P. (1976). The occurrence of visual neglect in patients with unilateral cerebral disease. Cortex 12, 221-231. doi: 10.1016/S0010-9452(76)80003-2

Compton, R. J., Heller, W., Banich, M. T., Palmieri, P. A., and Miller, G. A. (2000). Responding to threat: hemispheric asymmetries and interhemispheric division of input. Neuropsychology 14:254. doi: 10.1037//0894-4105.14.2.254

Corbetta, M., Patel, G., and Shulman, G. L. (2008). The reorienting system of the human brain: from environment to theory of mind. Neuron 58, 306-324. doi: 10.1016/j.neuron.2008.04.017

Costa, L. D., Vaughan, H. G. Jr., Horwitz, M., and Ritter, W. (1969). Patterns of behavioral deficit associated with visual spatial neglect. Cortex 5, 242-263. doi: 10.1016/S0010-9452(69)80033-X

Duncko, R., Cornwell, B., Cui, L., Merikangas, K. R., and Grillon, C. (2007). Acute exposure to stress improves performance in trace eyeblink conditioning and spatial learning tasks in healthy men. Learn. Mem. 14, 329-335. doi: 10.1101/ $\operatorname{lm} .483807$

Foti, F., Menghini, D., Petrosini, L., Valerio, G., Crinò, A., Vicari, S., et al. (2011). Spatial competences in Prader-Willi syndrome: a radial arm maze study. Behav. Genet. 41, 445-456. doi: 10.1007/s10519-011-9471-4

Foti, F., Sorrentino, P., Menghini, D., Montuori, S., Pesoli, M., Turriziani, P., et al. (2020). Peripersonal visuospatial abilities in williams syndrome analyzed by a table radial arm maze task. Front. Hum. Neurosci. 14:254. doi: 10.3389/fnhum. 2020.00254

Franceschini, C., Musetti, A., Zenesini, C., Palagini, L., Pelosi, A., Quattropani, M. C., et al. (2020). Poor quality of sleep and its consequences on mental health during COVID-19 lockdown in Italy. PsyArXiv [Preprint]. doi: 10.31234/osf.io/ ah6j3

Gehrman, P., Seelig, A. D., Jacobson, I. G., Boyko, E. J., Hooper, T. I., Gackstetter, G. D., et al. (2013). Predeployment. sleep duration and insomnia symptoms as risk factors for new-onset mental health disorders following military deployment. Sleep 36, 1009-1018

Gigliotta, O., Bartolomeo, P., and Miglino, O. (2015a). Approaching neuropsychological tasks through adaptive neurorobots. Conn. Sci. 27, 153-163. doi: 10.1080/09540091.2014.968094

Gigliotta, O., Bartolomeo, P., and Miglino, O. (2015b). Neuromodelling based on evolutionary robotics: on the importance of motor control for spatial attention. Cogn. Process. 16, 237-240. doi: 10.1007/s10339-015-0714-9

Gigliotta, O., Malkinson, T. S., Miglino, O., and Bartolomeo, P. (2017). Pseudoneglect in visual search: behavioral evidence and connectional constraints in simulated neural circuitry. eNeuro 4:ENEURO.0154-17.2017. doi: 10.1523/ENEURO.0154-17.2017

Gruzelier, J., and Phelan, M. (1991). Stress induced reversal of a lexical divided visual-field asymmetry accompanied by retarded electrodermal habituation. Int. J. Psychophysiol. 11, 269-276.

Jewell, G., and McCourt, M. E. (2000). Pseudoneglect: a review and meta-analysis of performance factors in line bisection tasks. Neuropsychologia 38, 93-110. doi: 10.1016/s0028-3932(99)00045-7

Klatzky, R. L. (1998). "Allocentric and egocentric spatial representations: definitions, distinctions, and interconnections," in Spatial Cognition. Lecture Notes in Computer Science, eds C. Freksa, C. Habel, and K. F. Wender (Heidelberg: Springer), 1-17.

Lazarus, R. S., and Folkman, S. (1984). Stress, Appraisal and Coping. New York, NY: Springer.

Lee, E., Hong, J., Park, Y.-G., Chae, S., Kim, Y., and Kim, D. (2015). Left brain cortical activity modulates stress effects on social behavior. Sci. Rep. 5:13342. doi: $10.1038 /$ srep 13342

Lindauer, R., Booij, J., Habraken, J., Van Meijel, E., Uylings, H., Olff, M., et al. (2008). Effects of psychotherapy on regional cerebral blood flow during trauma imagery in patients with post-traumatic stress disorder: a randomized clinical trial. Psychol. Med. 38, 543-554. doi: 10.1017/S003329170700 1432
Mandolesi, L., Addona, F., Foti, F., Menghini, D., Petrosini, L., and Vicari, S. (2009a). Spatial competences in Williams syndrome: a radial arm maze study. Int. J. Dev. Neurosci. 27, 205-213. doi: 10.1016/j.ijdevneu.2009.01.004

Mandolesi, L., Petrosini, L., Menghini, D., Addona, F., and Vicari, S. (2009b). Children's radial arm maze performance as a function of age and sex. Int. J. Dev. Neurosci. 27, 789-797. doi: 10.1016/j.ijdevneu.2009.08.010

Mandolesi, L., Polverino, A., Montuori, S., Foti, F., Ferraioli, G., Sorrentino, P., et al. (2018). Effects of physical exercise on cognitive functioning and wellbeing: biological and psychological benefits. Front. Psychol. 9:509. doi: 10.3389/fpsyg. 2018.00509

Matthews, G., and Campbell, S. E. (2009). Sustained performance under overload: personality and individual differences in stress and coping. Theor. Issues Ergon. Sci. 10, 417-442. doi: 10.1080/14639220903106395

Matthews, G., Davies, D. R., Stammers, R. B., and Westerman, S. J. (2000). Human Performance: Cognition, Stress, and Individual Differences. East Sussex, UK: Psychology Press.

Mundorf, A., Matsui, H., Ocklenburg, S., and Freund, N. (2020). Asymmetry of turning behavior in rats is modulated by early life stress. Behav. Brain. Res. 393:112807. doi: 10.1016/j.bbr.2020.112807

Newcombe, N. S. (2018). "Three kinds of spatial cognition," in Stevens' Handbook of Experimental Psychology and Cognitive Neuroscience, ed. J. T. Wixted (New York, NY: John Wiley \& Sons), 1-31.

Newcombe, N. S., and Huttenlocher, J. (2007). "Development of spatial cognition," in Handbook of Child Psychology, eds W. Damon, R. M. Lerner, D. Kuhn, and R. Siegler (New York, NY: John Wiley \& Sons).

Novotney, A. (2020). The risks of social isolation. Monit. Psychol. 50:5.

Ocklenburg, S., Korte, S. M., Peterburs, J., Wolf, O. T., and Güntürkün, O. (2016). Stress and laterality - The comparative perspective. Physiol. Behav. 164, 321-329. doi: 10.1016/j.physbeh.2016.06.020

Olton, D. S., and Samuelson, R. J. (1976). Remembrance of places passed: spatial memory in rats. J. Exp. Psychol. Anim. Behav. Process. 2:97. doi: 10.1037/00977403.2.2.97

Overman, W. H., Pate, B. J., Moore, K., and Peuster, A. (1996). Ontogeny of place learning in children as measured in the radial arm maze, Morris search task, and open field task. Behav. Neurosci. 110:1205. doi: 10.1037/0735-7044.110.6.1205

Pacella, D., Ponticorvo, M., Gigliotta, O., and Miglino, O. (2017). Basic emotions and adaptation. A computational and evolutionary model. PLoS One 12:e0187463. doi: 10.1371/journal.pone.0187463

Prunas, A., Sarno, I., Preti, E., and Madeddu, F. (2010). SCL-90-R. Symptom Checklist-90-R. Florence, IT: Giunti.

Raven, J. C., Raven, J. C., and Court, J. H. (1962). Advanced Progressive Matrices. London: HK Lewis.

Richardson, A. E., and VanderKaay Tomasulo, M. M. (2011). Influence of acute stress on spatial tasks in humans. Physiol. Behav. 103, 459-466. doi: 10.1016/j. physbeh.2011.03.019

Sampaio, E., and Chokron, S. (1992). Pseudoneglect and reversed pseudoneglect among left-handers and right-handers. Neuropsychologia 30, 797-805. doi: 10. 1016/0028-3932(92)90083-X

Santarnecchi, E., Sprugnoli, G., Tatti, E., Mencarelli, L., Neri, F., Momi, D., et al. (2018). Brain functional connectivity correlates of coping styles. Cogn. Affect. Behav. Neurosci. 18, 495-508. doi: 10.3758/s13415-018-0583-7

Schwabe, L., Oitzl, M. S., Philippsen, C., Richter, S., Bohringer, A., Wippich, W., et al. (2007). Stress modulates the use of spatial versus stimulus-response learning strategies in humans. Learn. Mem. 14, 109-116. doi: 10.1101/lm. 435807

Sica, C., Magni, C., Ghisi, M., Altoè, G., Sighinolfi, C., Chiri, L. R., et al. (2008). Coping orientation to the problems experienced-new Italian version (COPENVI). Psicoter. Cogn. Comportamentale. 14, 27-53.

Soares, J. M., Sampaio, A., Ferreira, L. M., Santos, N. C., Marques, P., Marques, F., et al. (2013). Stress impact on resting state brain networks. PLoS One 8:e66500. doi: 10.1371/journal.pone. 0066500

Sorrentino, P., Lardone, A., Pesoli, M., Liparoti, M., Montuori, S., Curcio, G., et al. (2019). The development of spatial memory analyzed by means of ecological walking task. Front. Psychol. 10:728. doi: 10.3389/fpsyg.2019. 00728

Sullivan, R. M. (2004). Hemispheric asymmetry in stress processing in rat prefrontal cortex and the role of mesocortical dopamine. Stress 7, 131-143. doi: $10.1080 / 102538900410001679310$ 
ter Horst, J. P., de Kloet, E. R., Schächinger, H., and Oitzl, M. S. (2012). Relevance of stress and female sex hormones for emotion and cognition. Cell. Mol. Neurobiol. 32, 725-735. doi: 10.1007/s10571-011-9774-2

Thiebaut de Schotten, M., Urbanski, M., Duffau, H., Volle, E., Lévy, R., Dubois, B., et al. (2005). Direct evidence for a parietal-frontal pathway subserving spatial awareness in humans. Science 309, 2226-2228. doi: 10.1126/science.111 6251

Toba, M.-N., Cavanagh, P., and Bartolomeo, P. (2011). Attention biases the perceived midpoint of horizontal lines. Neuropsychologia 49, 238-246. doi: 10.1016/j.neuropsychologia.2010.11.022

Vallortigara, G., and Versace, E. (2017). "Laterality at the neural, cognitive, and behavioral levels," in APA Handbook of Comparative Psychology: vol. 1. Basic Concepts, Methods, Neural Substrate, and Behavior, ed. J. Call (Washington, DC: American Psychological Association), 557-577.

van der Kooij, M. A., Jene, T., Treccani, G., Miederer, I., Hasch, A., Voelxen, N., et al. (2018). Chronic social stress-induced hyperglycemia in mice couples individual stress susceptibility to impaired spatial memory. Proc. Natl. Acad. Sci. U.S.A. 115, E10187-E10196. doi: 10.1073/pnas.1804412115

World Health Organization (2020). WHO Timeline - COVID-19. Statement. Geneva: WHO.

Zach, P., Valeš, K., Stuchlík, A., Čermáková, P., Mrzílková, J., Koutella, A., et al. (2016). Effect of stress on structural brain asymmetry. Neuroendocrinol. Lett. $37,253-264$.
Zurlo, M. C., Cattaneo Della Volta, M. F., and Vallone, F. (2019). Infertilityrelated stress and psychological health outcomes in infertile couples undergoing medical treatments: testing a multi-dimensional model. J. Clin. Psychol. Med. Settings. 1:15. doi: 10.1007/s10880-019-09653-z

Zurlo, M. C., Cattaneo Della Volta, M. F. C., and Vallone, F. (2020). COVID-19 student stress questionnaire: development and validation of a questionnaire to evaluate students' stressors related to the coronavirus pandemic lockdown. Front. Psychol. 11:576758. doi: 10.3389/fpsyg.2020.576758

Zurlo, M. C., Pes, D., and Capasso, R. (2013). Teacher stress questionnaire: validity and reliability study in Italy. Psychol. Rep. 113, 490-517. doi: 10.2466/03.16.PR0. $113 \times 23 z 9$

Conflict of Interest: The authors declare that the research was conducted in the absence of any commercial or financial relationships that could be construed as a potential conflict of interest.

Copyright (C) 2021 Somma, Bartolomeo, Vallone, Argiuolo, Cerrato, Miglino, Mandolesi, Zurlo and Gigliotta. This is an open-access article distributed under the terms of the Creative Commons Attribution License (CC BY). The use, distribution or reproduction in other forums is permitted, provided the original author(s) and the copyright owner(s) are credited and that the original publication in this journal is cited, in accordance with accepted academic practice. No use, distribution or reproduction is permitted which does not comply with these terms. 